\title{
Ring Opening and Expansion of Aziridines in a Silica-Water Reaction Medium
}

\author{
Supporting Information \\ Satoshi Minakata, * Takahiro Hotta, Yoji Oderaotoshi, and Mitsuo Komatsu* \\ Department of Applied Chemistry, Graduate School of Engineering, Osaka University, \\ Yamadaoka 2-1, Suita, Osaka 565-0871, Japan \\ minakata@chem.eng.osaka-u.ac.jp
}

\section{Table of Contents}

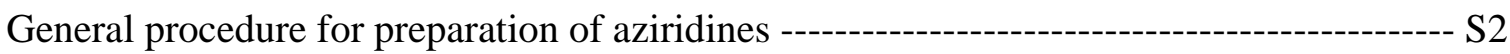

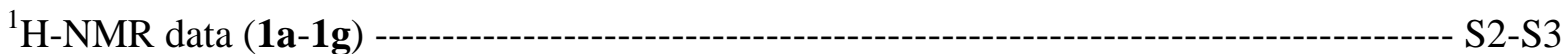

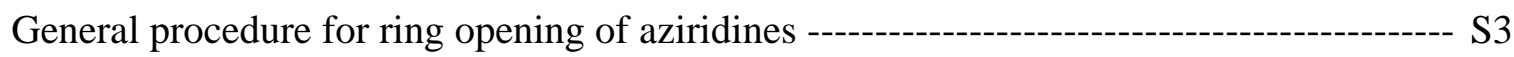

Spectral data (2a-2f, 3a+3b, 4a) -

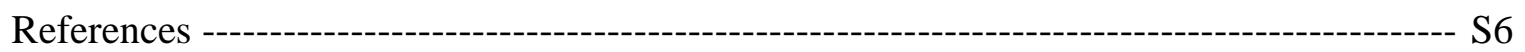

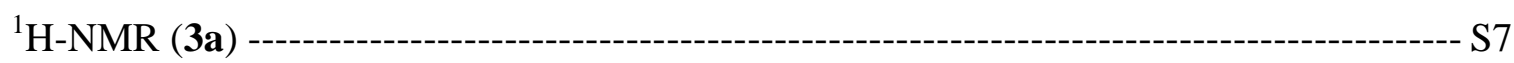

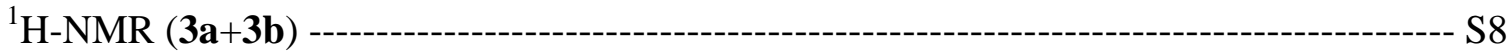

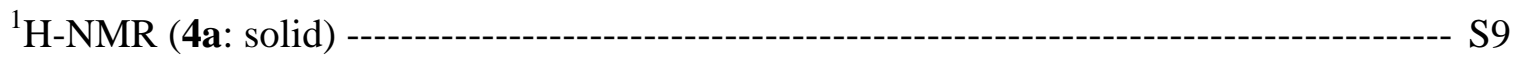

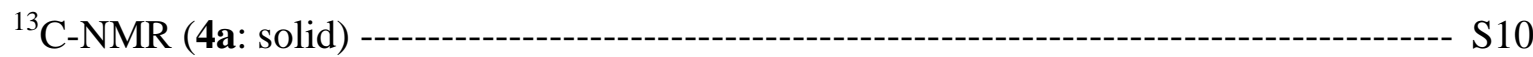

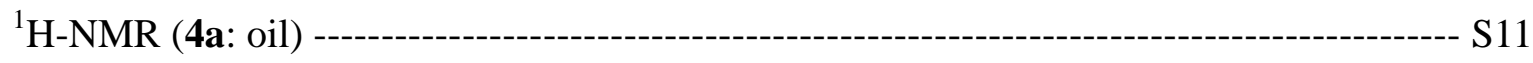

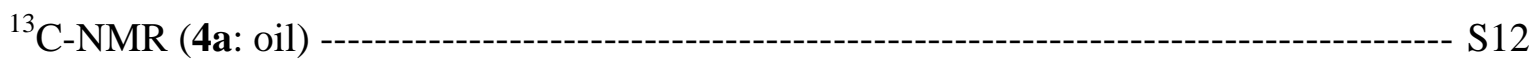


Materials. (a general procedure for preparation of aziridines according to our method ${ }^{1}$ )

The alkenes $(2.0 \mathrm{mmol})$ were added to a solution of iodine $(0.1 \mathrm{mmol}, 10$ mol\%), Chloramine-T $(1.0 \mathrm{mmol})$ in distilled MeCN $(3.0 \mathrm{~mL})$. The solution was stirred at room temperature under a nitrogen atmosphere. After the addition of $\mathrm{CH}_{2} \mathrm{Cl}_{2}(40 \mathrm{~mL})$ and water $(20 \mathrm{~mL})$, the phases were separated and aqueous phase was extracted with $\mathrm{CH}_{2} \mathrm{Cl}_{2}(20$ $\mathrm{mL})$. The combined organic extracts were washed with water (20 $\mathrm{mL} \mathrm{x} \mathrm{3)}$ and brine $(20 \mathrm{~mL})$, dried over $\mathrm{K}_{2} \mathrm{CO}_{3}$ and concentrated to give the crude product. Purification by flash column chromatography (silica gel, EtOAc in hexane) gave the aziridines. Further purification was performed by recycling preparative HPLC (GPC column, chloroform), if necessary.

$\boldsymbol{N}$-(p-Toluenesulfonyl)-2-n-hexylaziridine (1a) $)^{2}$ : Colorless oil; ${ }^{1} \mathrm{H}$ NMR $\left(270 \mathrm{MHz}, \mathrm{CDCl}_{3}\right) \delta=$ 0.86 (t, 3H, $J=7.3$ Hz), 1.20-1.33 (m, 10H), 2.05 (d, 1H, $J=4.6$ Hz), 2.44 (s, 3H,), 2.62-2.74 (m, 2H), 7.32 (d, 2H, $J=8.1 \mathrm{~Hz}), 7.82(\mathrm{~d}, 2 \mathrm{H}, J=8.1 \mathrm{~Hz})$

$\boldsymbol{N}$-(p-Toluenesulfonyl)-2-benzylaziridine (1b) ${ }^{3}$ : White solid; Mp: 94-95 ${ }^{\circ} \mathrm{C}$; ${ }^{1} \mathrm{H}$ NMR (270 $\left.\mathrm{MHz}, \mathrm{CDCl}_{3}\right) \delta=2.16$ (d, 1H, $J=4.3 \mathrm{~Hz}$ ), 2.42 (s, 3H), 2.64-2.80 (m, 2H), 2.82-2.99 (m, 2H), 7.01-7.22 (m, 7H), 7.66 (d, 2H, $J=8.4 \mathrm{~Hz})$

$\boldsymbol{N}$-(p-Toluenesulfonyl)-2-sec-butylaziridine (1c) ${ }^{4}$ as a mixture of diastereomers: Colorless oil; ${ }^{1} \mathrm{H}$ NMR (270 MHz, $\mathrm{CDCl}_{3}$ ) $\delta=0.75-1.40$ (m, 9H), 2.09 (dd, $1 \mathrm{H}, J=3.2,13.2 \mathrm{~Hz}$ ), 2.45-2.67 (m, $5 \mathrm{H}), 7.32$ (d, $2 \mathrm{H}, J=8.1 \mathrm{~Hz}), 7.81(\mathrm{~d}, 2 \mathrm{H}, J=8.1 \mathrm{~Hz})$

$N$-(p-Toluenesulfonyl)-2-phenylaziridine (1d) $)^{5}$ : White solid; Mp: $92-93{ }^{\circ} \mathrm{C}$; ${ }^{1} \mathrm{H}$ NMR (270 $\left.\mathrm{MHz}, \mathrm{CDCl}_{3}\right) \delta=2.38$ (d, 1H, $J=4.3 \mathrm{~Hz}$ ), 2.44 (s, 3H), 2.97 (d, 1H, $J=7.3 \mathrm{~Hz}$ ), 3.75 (dd, 1H, $J$ = 4.3, 7.3 Hz), 7.19-7.34 (m, 7H), 7.85 (d, 2H, $J=8.4 \mathrm{~Hz})$

$\boldsymbol{N}$-(p-Toluenesulfonyl)-2-hydroxymethylaziridine $\quad(\mathbf{1 e})^{4}$ : Colorless oil; ${ }^{1} \mathrm{H} \quad \mathrm{NMR} \quad(270$ $\left.\mathrm{MHz}, \mathrm{CDCl}_{3}\right) \delta=2.00$ (br s, $1 \mathrm{H}$ ), 2.31 (d, $1 \mathrm{H}, J=4.6 \mathrm{~Hz}$ ), 2.45 (s, 3H), 2.61 (d, $1 \mathrm{H}, J=7.0 \mathrm{~Hz}$ ), 2.98-3.06 (m, 1H), 3.50-3.59 (m, 1H), 3.80-3.87 (m, 1H), 7.33 (d, 2H, $J=8.4 \mathrm{~Hz}$ ), 7.81 (d, 2H, $J$ $=8.4 \mathrm{~Hz})$

1,2-[N-(p-Toluenesulfonyl)imino]cyclopentane (1f) ${ }^{6}$ : White solid; Mp: 61-62 ${ }^{\circ} \mathrm{C} ;{ }^{1} \mathrm{H}$ NMR (270 
$\left.\mathrm{MHz}, \mathrm{CDCl}_{3}\right) \delta=1.37-2.16$ (m, 6H), 2.44 (s, 3H), 3.33 (s, 2H), 7.33 (d, 2H, $J=8.4 \mathrm{~Hz}$ ), 7.79 (d, $2 \mathrm{H}, J=8.4 \mathrm{~Hz}$ )

1,2-[N-(p-Toluenesulfonyl)imino]cyclohexane (1g) $)^{5}$ : White solid; $\mathrm{Mp}: 55-56{ }^{\circ} \mathrm{C} ; \quad{ }^{1} \mathrm{H}$ NMR $\left(270 \mathrm{MHz}, \mathrm{CDCl}_{3}\right) \delta=1.20-1.81(\mathrm{~m}, 8 \mathrm{H}), 2.44$ (s, 3H), 2.97 (t, 2H, $\left.J=1.4 \mathrm{~Hz}\right), 7.30$ (d, 2H, $J=$ $8.1 \mathrm{~Hz}), 7.80$ (d, $2 \mathrm{H}, J=8.1 \mathrm{~Hz})$

\section{General Procedure for Ring Opening of Aziridines.}

To a mixture of potassium cyanide (65 mg, $1 \mathrm{mmol}$ ) and Silica Gel 60 (0.5 g) in distilled water $(1.0 \mathrm{~mL})$ aziridine 1a $(141 \mathrm{mg}, 0.5 \mathrm{mmol})$ was added. The mixture was stirred at $80{ }^{\circ} \mathrm{C}$ for $24 \mathrm{~h}$ under an ambient atmosphere. After the addition of acetone $(5 \mathrm{~mL})$, the mixture was passed through a short celite column using acetone $(10 \mathrm{~mL})$ and $\mathrm{Et}_{2} \mathrm{O}(10 \mathrm{~mL})$ as eluents. The filtrate was dried over $\mathrm{Na}_{2} \mathrm{SO}_{4}$ and concentrated to give the crude product. Purification by flash column chromatography (silica gel; 10\% ethyl acetate in hexane) gave $107 \mathrm{mg}$ (69\%) of ring-opening product $\mathbf{2 a}$ as colorless oil. The characteristics of products in this paper (2a-g, 3a) were found to be identical to those published previously. ${ }^{4,7}$

$N$-(2-Cyanooctyl)-4-methylbenzenesulfonamide (2a) ${ }^{7}$ : Colorless oil; IR (neat, $\mathrm{cm}^{-1}$ ) 2251 $(\mathrm{CN}) ;{ }^{1} \mathrm{H}$ NMR $\left(270 \mathrm{MHz}, \mathrm{CDCl}_{3}\right) \delta=0.84$ (t, 3H, $\left.J=6.8 \mathrm{~Hz}\right), 1.07-1.60$ (m, 10H), 2.44 (s, 3H), 2.50-2.68 (m, 2H), 3.38-3.46 (m, 1H), 4.75 (d, 1H, $J=7.6 \mathrm{~Hz}), 7.32$ (d, 2H, $J=8.2 \mathrm{~Hz}$ ), 7.75 (d, $2 \mathrm{H}, J=8.2 \mathrm{~Hz}) ;{ }^{13} \mathrm{C} \mathrm{NMR}\left(\mathrm{CDCl}_{3}\right) \delta=14.1,21.6,22.5,25.1,25.3,28.5,31.5,33.9,50.0,116.7$, 127.0, 129.8, 136.9, 143.8; MS (CI, methane) $\mathrm{m} / \mathrm{z}$ (relative intensity, \%) 309 ([M+1] $]^{+}, 61$ ), 268 ([M-CH $2 \mathrm{CN}]^{+}, 100$ ); HRMS (CI, methane) $\mathrm{m} / \mathrm{z}$ Calcd for $\mathrm{C}_{16} \mathrm{H}_{25} \mathrm{~N}_{2} \mathrm{O}_{2} \mathrm{~S}(\mathrm{M}+\mathrm{H})$ 309.1637, found 309.1646; Anal. Calcd for $\mathrm{C}_{16} \mathrm{H}_{24} \mathrm{~N}_{2} \mathrm{O}_{2} \mathrm{~S}$ : C, 62.30; H, 7.84; N, 9.08. Found: C, 61.89; H, 7.52; N, 8.92.

$\boldsymbol{N}$-(2-Cyano-1-benzylethyl)-4-methylbenzenesulfonamide (2b) ${ }^{4}$ : White solid; Mp: 102-103 ${ }^{\circ} \mathrm{C}$; IR (neat, $\mathrm{cm}^{-1}$ ) $2251(\mathrm{CN}) ;{ }^{1} \mathrm{H}$ NMR (270 MHz, $\left.\mathrm{CDCl}_{3}\right) \delta=2.42$ (s, 3H), 2.51-2.58 (dd, $1 \mathrm{H}, J$ = 3.8, $16.7 \mathrm{~Hz}$ ), 2.62-2.70 (dd, $1 \mathrm{H}, J=6.5,16.7 \mathrm{~Hz}$ ), 2.73-2.81 (dd, $1 \mathrm{H}, J=7.8,13.9 \mathrm{~Hz}$ ), 2.86-2.93 (dd, 1H, $J=6.8,13.9 \mathrm{~Hz}$ ), 3.60-3.67 (m, 1H), 4.96 (br s, 1H), 6.99 (d, 2H, $J=8.4 \mathrm{~Hz}$ ), 7.18-7.22 (m, 5H), 7.54 (d, 2H, $J=8.4 \mathrm{~Hz}) ;{ }^{13} \mathrm{C} \mathrm{NMR}\left(\mathrm{CDCl}_{3}\right) \delta=21.6,24.2,39.8,51.4,116.7$, 126.8, 127.2, 128.3, 128.9, 129.7, 134.9, 136.2, 143.7; MS (CI, methane) $\mathrm{m} / \mathrm{z}$ 
(relative intensity, \%) $315\left([\mathrm{M}+1]^{+}, 100\right), 274\left(\left[\mathrm{M}-\mathrm{CH}_{2} \mathrm{CN}\right]^{+}, 100\right)$; HRMS (CI, methane) $\mathrm{m} / \mathrm{z}$ Calcd for $\mathrm{C}_{17} \mathrm{H}_{19} \mathrm{~N}_{2} \mathrm{O}_{2} \mathrm{~S}(\mathrm{M}+\mathrm{H})$ 315.1168, found 315.1154. Anal. Calcd for $\mathrm{C}_{17} \mathrm{H}_{18} \mathrm{~N}_{2} \mathrm{O}_{2} \mathrm{~S}$ : C, 64.94; H, 5.77; N, 8.91. Found: C, 64.65; H, 5.76; N, 8.73.

$\mathrm{N}$-(1-Cyanomethyl-2-methylbutyl)-4-methylbenzenesulfonamide (2c) ${ }^{4}$ as a mixture of diastereomers: Colorless oil; IR (neat, $\mathrm{cm}^{-1}$ ) 2251 (CN); ${ }^{1} \mathrm{H}$ NMR (270 MHz, $\left.\mathrm{CDCl}_{3}\right) \delta=$ 0.72-1.67 (m, 9H), 2.44 (s, 3H), 2.55-2.63 (m, 2H), 3.25-3.43 (m, 1H), 4.72 (br s, 1H), 7.32 (d, $2 \mathrm{H}, J=8.4 \mathrm{~Hz}$ ), 7.78 (d, $2 \mathrm{H}, J=8.4 \mathrm{~Hz}) ;{ }^{13} \mathrm{C} \mathrm{NMR}\left(\mathrm{CDCl}_{3}\right) \delta=15.3,21.7,22.3,24.5,37.3$, 37.6, 53.4, 116.7, 127.0, 129.8, 136.9, 144.0; MS (CI, methane) $\mathrm{m} / \mathrm{z}$ (relative intensity, \%) 281 ([M+1] $\left.]^{+}, 37\right), 240$ ([M-CH $\left.\left.\mathrm{CH}_{2} \mathrm{CN}\right]^{+}, 100\right)$; HRMS (CI, methane) $\mathrm{m} / \mathrm{z}$ Calcd for $\mathrm{C}_{14} \mathrm{H}_{21} \mathrm{~N}_{2} \mathrm{O}_{2} \mathrm{~S}$ $(\mathrm{M}+\mathrm{H})$ 281.1324, found 281.1318; Anal. Calcd for $\mathrm{C}_{14} \mathrm{H}_{20} \mathrm{~N}_{2} \mathrm{O}_{2} \mathrm{~S}$ : C, 59.97; H, 7.19; N, 9.99. Found: C, 59.69; H, 6.94; N, 9.86.

N-(2-Cyano-1-phenylethyl)-4-methylbenzenesulfonamide (2d) ${ }^{7}$ : White solid; Mp: 120-121 ${ }^{\circ} \mathrm{C}$; IR (neat, $\mathrm{cm}^{-1}$ ) $2249(\mathrm{CN}) ;{ }^{1} \mathrm{H}$ NMR $\left(270 \mathrm{MHz}, \mathrm{CDCl}_{3}\right) \delta=2.42$ (s, 3H), 2.93 (ddd, $2 \mathrm{H}, J=$ 5.4, 7.0, $16.7 \mathrm{~Hz}), 4.54-4.59$ (m, 1H), 5.09 (d, 1H, $J=6.8 \mathrm{~Hz}), 7.12$ (d, 2H, $J=8.2 \mathrm{~Hz}$ ), 7.24-7.31 (m, 5H), 7.68 (d, 2H, $J=8.2 \mathrm{~Hz}) ;{ }^{13} \mathrm{C} \mathrm{NMR}\left(\mathrm{CDCl}_{3}\right) \delta=21.5,26.3,54.1,116.4,126.2$, 127.1, 128.9, 129.2, 129.8, 136.4, 137.1, 144.0; MS (CI, methane) $\mathrm{m} / \mathrm{z}$ (relative intensity, \%) 301 ([M+1] $\left.]^{+}, 100\right), 260$ ([M-CH$\left.{ }_{2} \mathrm{CN}\right]^{+}$, 43); HRMS (CI, methane) $\mathrm{m} / \mathrm{z}$ Calcd for $\mathrm{C}_{16} \mathrm{H}_{17} \mathrm{~N}_{2} \mathrm{O}_{2} \mathrm{~S}(\mathrm{M}+\mathrm{H})$ 301.1011, found 301.1008. Anal. Calcd for $\mathrm{C}_{16} \mathrm{H}_{16} \mathrm{~N}_{2} \mathrm{O}_{2} \mathrm{~S}$ : C, 63.98; H, 5.37; N, 9.33. Found: C, 63.70; H, 5.32; N, 9.21.

$\mathrm{N}$-(2-Cyano-1-hydroxymethylethyl)-4-methylbenzenesulfonamide (2e) ${ }^{4}$ : Colorless oil; IR (neat, $\mathrm{cm}^{-1}$ ) $2252(\mathrm{CN}) ;{ }^{1} \mathrm{H}$ NMR (270 MHz, $\left.\mathrm{CDCl}_{3}\right) \delta=2.44(\mathrm{~s}, 3 \mathrm{H}), 2.62-2.66(\mathrm{~m}, 3 \mathrm{H})$, 3.61-3.67 (m, 3H), 5.66 (d, $1 \mathrm{H}, J=7.6 \mathrm{~Hz}), 7.32$ (d, 2H, $J=8.4 \mathrm{~Hz}), 7.78$ (d, 2H, $J=8.4 \mathrm{~Hz}$ ); ${ }^{13} \mathrm{C}$ NMR $\left(\mathrm{CDCl}_{3}\right) \delta=21.0,21.7,51.1,62.6,116.7,127.0,129.9,136.6,144.1$; MS (CI, methane) $\mathrm{m} / \mathrm{z}$ (relative intensity, \%) $255\left([\mathrm{M}+1]^{+}, 100\right), 223\left(\left[\mathrm{M}-\mathrm{CH}_{2} \mathrm{OH}\right]^{+}, 25\right)$; HRMS (CI, methane) $\mathrm{m} / \mathrm{z}$ Calcd for $\mathrm{C}_{11} \mathrm{H}_{15} \mathrm{~N}_{2} \mathrm{O}_{3} \mathrm{~S}(\mathrm{M}+\mathrm{H})$ 255.0804, Found 255.0806.

$\mathrm{N}$-(2-Cyanocyclopentyl)-4-methylbenzenesulfonamide (2f) ${ }^{7}$ : White solid; Mp: 107-108 ${ }^{\circ} \mathrm{C}$; IR (neat, $\mathrm{cm}^{-1}$ ) $2243(\mathrm{CN}) ;{ }^{1} \mathrm{H}$ NMR (270 MHz, $\mathrm{CDCl}_{3}$ ) $\delta=1.44-2.11$ (m, 6H), 2.43 (s, 3H), 2.83 (ddd, $1 \mathrm{H}, J=5.9,7.3,10.5 \mathrm{~Hz}$ ), 3.74-3.78 (m, 1H), 5.56 (br s, $1 \mathrm{H}$ ), 7.36 (d, 2H, $J=8.4 \mathrm{~Hz}$ ), 7.80 
(d, $2 \mathrm{H}, J=8.4 \mathrm{~Hz}) ;{ }^{13} \mathrm{C}$ NMR $\left(\mathrm{CDCl}_{3}\right) \delta=21.7,22.7,29.0,32.7,35.7,58.6,121.0,127.1,129.9$, 136.3, 144.0; MS (CI, methane) $\mathrm{m} / \mathrm{z}$ (relative intensity, \%) $265\left([\mathrm{M}+1]^{+}, 100\right), 172\left(\left[\mathrm{M}-\mathrm{CH}_{3} \mathrm{Ar}\right]^{+}\right.$, 22); HRMS (CI, methane) $\mathrm{m} / \mathrm{z}$ Calcd for $\mathrm{C}_{13} \mathrm{H}_{17} \mathrm{~N}_{2} \mathrm{O}_{2} \mathrm{~S}$ (M+H) 265.1011, found 265.1004; Anal. Calcd for $\mathrm{C}_{13} \mathrm{H}_{16} \mathrm{~N}_{2} \mathrm{O}_{2} \mathrm{~S}$ : C, 59.07; H, 6.10; N, 10.60. Found: C, 58.80; H, 6.08; N, 10.43 .

$N$-(2-Cyanocyclohexyl)-4-methylbenzenesulfonamide (2g) ${ }^{7}$ : White solid; Mp: $115-116{ }^{\circ} \mathrm{C}$; IR (neat, $\mathrm{cm}^{-1}$ ) $2245(\mathrm{CN}) ;{ }^{1} \mathrm{H}$ NMR (270 MHz, $\left.\mathrm{CDCl}_{3}\right) \delta=1.24-2.05$ (m, 8H), 2.44 (s, 3H), 2.65 (ddd, 1H, $J=3.8,8.1,8.9 \mathrm{~Hz}$ ), 3.35-3.39 (m, 1H), 5.18 (br s, 1H), 7.33 (d, 2H, $J=8.4 \mathrm{~Hz}$ ), 7.81 $(\mathrm{d}, 2 \mathrm{H}, J=8.4 \mathrm{~Hz}) ;{ }^{13} \mathrm{C} \mathrm{NMR}\left(\mathrm{CDCl}_{3}\right) \delta=21.7,22.6,23.0,27.3$, 31.4, 34.6, 52.7, 120.2, 127.1, 129.7, 137.0, 143.8; MS (CI, methane) m/z (relative intensity, \%) 279 ([M+1] $\left.]^{+}, 100\right), 125$ ([M- $\left.\mathrm{CH}_{3} \mathrm{ArSO}_{2}\right]^{+}$, 29); HRMS (CI, methane) $\mathrm{m} / \mathrm{z}$ Calcd for $\mathrm{C}_{14} \mathrm{H}_{19} \mathrm{~N}_{2} \mathrm{O}_{2} \mathrm{~S}(\mathrm{M}+\mathrm{H})$ 279.1167, Found 279.1159.

$N$-(2-Iodooctyl)-4-methylbenzenesulfonamide (3a) and $N$-(1-Iodooctyl)-4-methylbenzenesulfonamide (3b) as an inseparable 94:6 in 96\% yield. : Colorless oil; IR $\left(\mathrm{CHCl}_{3}, \mathrm{~cm}^{-1}\right)(3 \mathbf{a}+3 \mathbf{b}) 1161 ;{ }^{1} \mathrm{H} \mathrm{NMR}$ $\left(270 \mathrm{MHz}, \mathrm{CDCl}_{3}\right)(3 \mathbf{a}+\mathbf{3 b}) \delta=0.85(\mathrm{t}, 3 \mathrm{H}, J=6.8 \mathrm{~Hz}, 3 \mathbf{a}), 0.88(\mathrm{t}, 3 \mathrm{H}, J=6.5 \mathrm{~Hz}, 3 \mathbf{b})$, 1.13-1.42 (m, 18H, 10H of 3a and 8H of 3b), $1.73(\mathrm{~m}, 2 \mathrm{H}, 3 \mathbf{b}), 2.43(\mathrm{~s}, 6 \mathrm{H}, 3 \mathrm{H}$ of $3 \mathbf{a}$ and $3 \mathrm{H}$ of 3b), 2.94 (m, 1H, 3a), 3.18 (dd, 1H, $J=5.4,10.4$ Hz, 3a), 3.25 (dd, 1H, $J=7.3,10.4$ Hz, 3a), 3.24-3.34 (m, 2H, 3b), 4.02 (m, 1H, 3b), 4.67 (d, 1H, $J=8.6 \mathrm{~Hz}, 3 \mathbf{a}), 4.91$ (t, 1H, $J=6.5 \mathrm{~Hz}, 3 \mathbf{b}$ ), 7.31 (d, 2H, $J=8.4 \mathrm{~Hz}, 3 \mathbf{a}), 7.32$ (d, 2H, $J=8.4 \mathrm{~Hz}, 3 \mathbf{b}$ ), 7.75 (d, 2H, $J=8.4 \mathrm{~Hz}, 3 \mathbf{b}$ ), 7.77 (d, $2 \mathrm{H}, J=8.4 \mathrm{~Hz}, 3 \mathbf{a}) ;{ }^{13} \mathrm{C} \mathrm{NMR}\left(\mathrm{CDCl}_{3}\right)(\mathbf{3 a}+\mathbf{3 b}) \delta=14.0,14.9,21.5,22.4,22.5$, 25.1, 28.3, 28.6, 29.14, 31.50, 31.51, 35.54, 36.0, 37.2, 50.9, 52.4, 127.0, 129.7, 129.8, 136.8, 137.7, 143.6, 143.8; MS (CI, isobutane): m/z (relative intensity, \%) (3a): 410 ([M+1] $\left.]^{+}, 30\right), 282$ ([M-I $\left.]^{+}, 52\right), 155$ ([ArSO $], 100),(3 \mathbf{b}): 410$ ([M+1] $\left.]^{+}, 17\right), 282$ ([M-I] $\left.]^{+}, 67\right), 155$ ([ArSO $\left.], 100\right) ; H R M S(C I$, methane) (3a); $\mathrm{m} / \mathrm{z}$ Calcd for $\mathrm{C}_{15} \mathrm{H}_{25} \mathrm{INO}_{2} \mathrm{~S}(\mathrm{M}+\mathrm{H})$ 410.0651, found 410.0626; (3b): $\mathrm{m} / \mathrm{z}$ Calcd for $\mathrm{C}_{15} \mathrm{H}_{25} \mathrm{INO}_{2} \mathrm{~S}(\mathrm{M}+\mathrm{H})$ 410.0651, Found 410.0642.

Although compound 3a could be isolated, $3 \mathbf{b}$ could not be separated. The charts of ${ }^{1} \mathrm{H}-\mathrm{NMR}$ of 3a and ca. 1:1 mixture of $\mathbf{3 a}$ and $3 \mathbf{b}$ were attached.

\section{4-Hexenyl-3-(p-toluenesulfonyl)-2-\{ $N$-(p-toluenesulfonyl)-2-aminooctyl $\}$ thiazolidine (4a)}


The products were obtained as two diastereomers which are separable but complete stereochemistry was not determined.

- White solid; Mp: 58-62 ${ }^{\circ} \mathrm{C}$; IR $\left(\mathrm{CHCl}_{3}, \mathrm{~cm}^{-1}\right) 1651(\mathrm{C}=\mathrm{N}) ;{ }^{1} \mathrm{H}$ NMR $\left(270 \mathrm{MHz}, \mathrm{CDCl}_{3}\right) \delta=$ 0.86 (t, 3H, $J=7.0 \mathrm{~Hz}), 0.91(\mathrm{t}, 3 \mathrm{H}, J=6.5 \mathrm{~Hz}), 1.12-1.43$ (m, 18H), 1.87 (dt, $2 \mathrm{H}, J=6.8 \mathrm{~Hz})$, 2.41 (s, 3H), 2.45 (s, 3H), 2.78 (1H, dd, $J=3.0,13.5 \mathrm{~Hz}$ ), 2.85 (1H, dd, $J=4.0,13.5 \mathrm{~Hz}$ ), 2.97 (d, $1 \mathrm{H}, J=11.1 \mathrm{~Hz}$ ), 3.23 (m, 1H), 3.41 (dd, 1H, $J=6.8,11.1 \mathrm{~Hz}$ ), 4.53 (d, 1H, $J=8.9 \mathrm{~Hz}$ ), 4.81 (dt, $1 \mathrm{H}, J=6.8 \mathrm{~Hz}$ ), 7.24 (d, 2H, $J=8.1 \mathrm{~Hz}), 7.35$ (d, 2H, $J=8.1 \mathrm{~Hz}), 7.64$ (d, 2H, $J=8.1 \mathrm{~Hz}), 7.81$ $(\mathrm{d}, 2 \mathrm{H}, J=8.1 \mathrm{~Hz}) ;{ }^{13} \mathrm{C} \mathrm{NMR}\left(\mathrm{CDCl}_{3}\right) \delta=14.1,21.5,21.6,22.5,25.6,26.0$, 28.9, 31.7, 31.9, 33.2, 33.3, 53.6, 57.4, 61.1, 126.9, 128.1, 129.4, 129.6, 136.9, 138.5, 142.9, 144.6, 152.9; MS (CI, methane): $m / z$ (relative intensity, \%): $622\left([\mathrm{M}+1]^{+}, 100\right), 468\left(\left[\mathrm{M}_{-} \mathrm{C}_{6} \mathrm{H}_{13} \mathrm{CH}(\mathrm{NH}) \mathrm{CH}_{2} \mathrm{~N}=\mathrm{C}\right]^{+}\right.$, 68); HRMS (CI, methane) $\mathrm{m} / \mathrm{z}$ Calcd for $\mathrm{C}_{31} \mathrm{H}_{48} \mathrm{~N}_{3} \mathrm{O}_{4} \mathrm{~S}_{3}(\mathrm{M}+\mathrm{H})$ 622. 2807, Found 622.2814.

- Colorless oil; IR $\left(\mathrm{CHCl}_{3}, \mathrm{~cm}^{-1}\right) 1651(\mathrm{C}=\mathrm{N}) ;{ }^{1} \mathrm{H}$ NMR $\left(600 \mathrm{MHz}, \mathrm{CDCl}_{3}\right) \delta=0.86$ (t, 3H, $J=$ $7.2 \mathrm{~Hz}$ ), 0.89 (t, 3H, $J=6.6 \mathrm{~Hz}), 1.14-1.41(\mathrm{~m}, 18 \mathrm{H}), 1.84$ (dt, 2H, $J=6.6 \mathrm{~Hz}), 2.40(\mathrm{~s}, 3 \mathrm{H})$, 2.46 (s, 3H), 2.81 (1H, dd, $J=4.2,13.2 \mathrm{~Hz}), 2.91(1 \mathrm{H}, \mathrm{dd}, J=1.8,13.2 \mathrm{~Hz}), 2.95$ (d, 1H, $J=$ $11.4 \mathrm{~Hz}$ ), 3.28 (m, 1H), 3.36 (dd, 1H, $J=6.6,11.4 \mathrm{~Hz}$ ), 4.35 (d, 1H, $J=9.0 \mathrm{~Hz}$ ), 4.80 (dt, $1 \mathrm{H}, J=$ $6.6 \mathrm{~Hz}$ ), 7.25 (d, 2H, $J=8.4 \mathrm{~Hz}$ ), 7.35 (d, 2H, $J=8.4 \mathrm{~Hz}$ ), 7.64 (d, 2H, $J=8.4 \mathrm{~Hz}$ ), 7.82 (d, $2 \mathrm{H}, J=8.4 \mathrm{~Hz}) ;{ }^{13} \mathrm{C} \mathrm{NMR}\left(\mathrm{CDCl}_{3}\right) \delta=14.15,14.18,21.6,21.7,22.60,22.63,25.8,26.0,28.97$, 29.03, 31.7, 31.8, 32.0, 33.3, 33.6, 53.8, 57.7, 61.2, 126.8, 128.0, 129.3, 129.5, 136.8, 138.5, 142.7, 144.6, 153.2; MS (CI, isobutane) : $\mathrm{m} / \mathrm{z}$ (relative intensity): $622\left([\mathrm{M}+1]^{+}, 100\right), 468$ ([M- $\left.\mathrm{C}_{6} \mathrm{H}_{13} \mathrm{CH}(\mathrm{NH}) \mathrm{CH}_{2} \mathrm{~N}=\mathrm{C}\right]^{+}, 12$ ); HRMS (CI, methane) $\mathrm{m} / \mathrm{z}$ Calcd for $\mathrm{C}_{31} \mathrm{H}_{48} \mathrm{~N}_{3} \mathrm{O}_{4} \mathrm{~S}_{3}(\mathrm{M}+\mathrm{H}$ ) 622. 2807, Found 622.2813.

\section{References}

1. Ando, T.; Kano, D.; Minakata, S.; Ryu, I.; Komatsu, M. Tetrahedron 1998, 54, 13485-13494.

2. Toshima, A.; Abe, H.; Hirosawa, C.; Tamao, K. J. Chem. Soc. Perkin Trans. 1, 1994, 3465-3471.

3. Alonso, D. A.; Andersson, P. G. J. Org. Chem. 1998, 63, 9455-9461.

4. Minakata, S.; Okada, Y.; Oderaotoshi, Y.; Komatsu, M. Org. Lett. 2005, 7, 3509-3512.

5. Evans, D. A.; Faul, M. M.; Bilodeau, M. T. J. Am. Chem. Soc. 1994, 116, 2742-2753.

6. Jeong, J. U.; Tao, B.; Sagasser, I.; Henniges, H.; Sharpless, K. B. J. Am. Chem. Soc. 1998, 120, 6844-6845.

7. Wu, J.; Hou, X.-L.; Dai, L.-X. J. Org. Chem. 2000, 65, 1344-1348. 


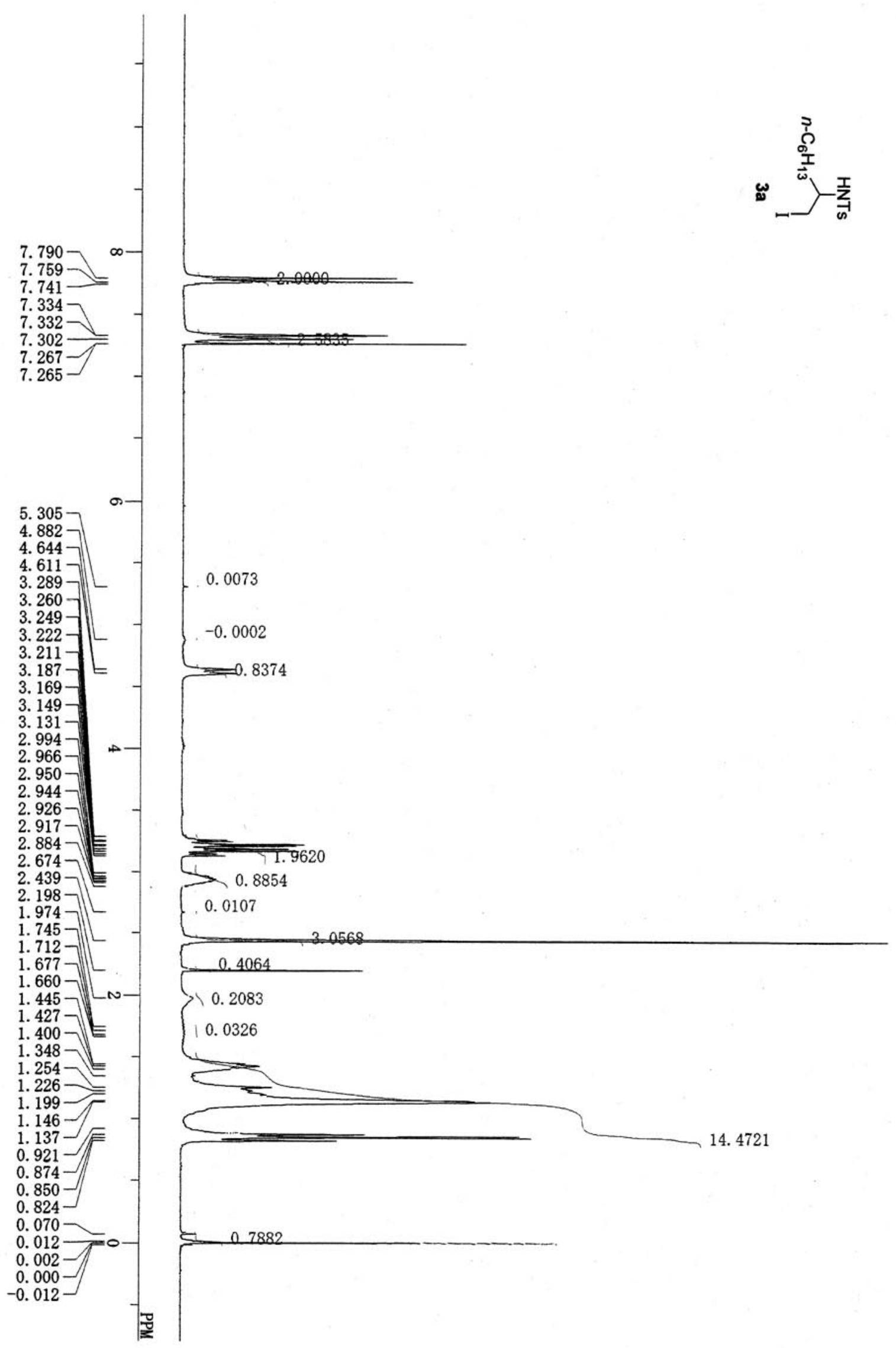




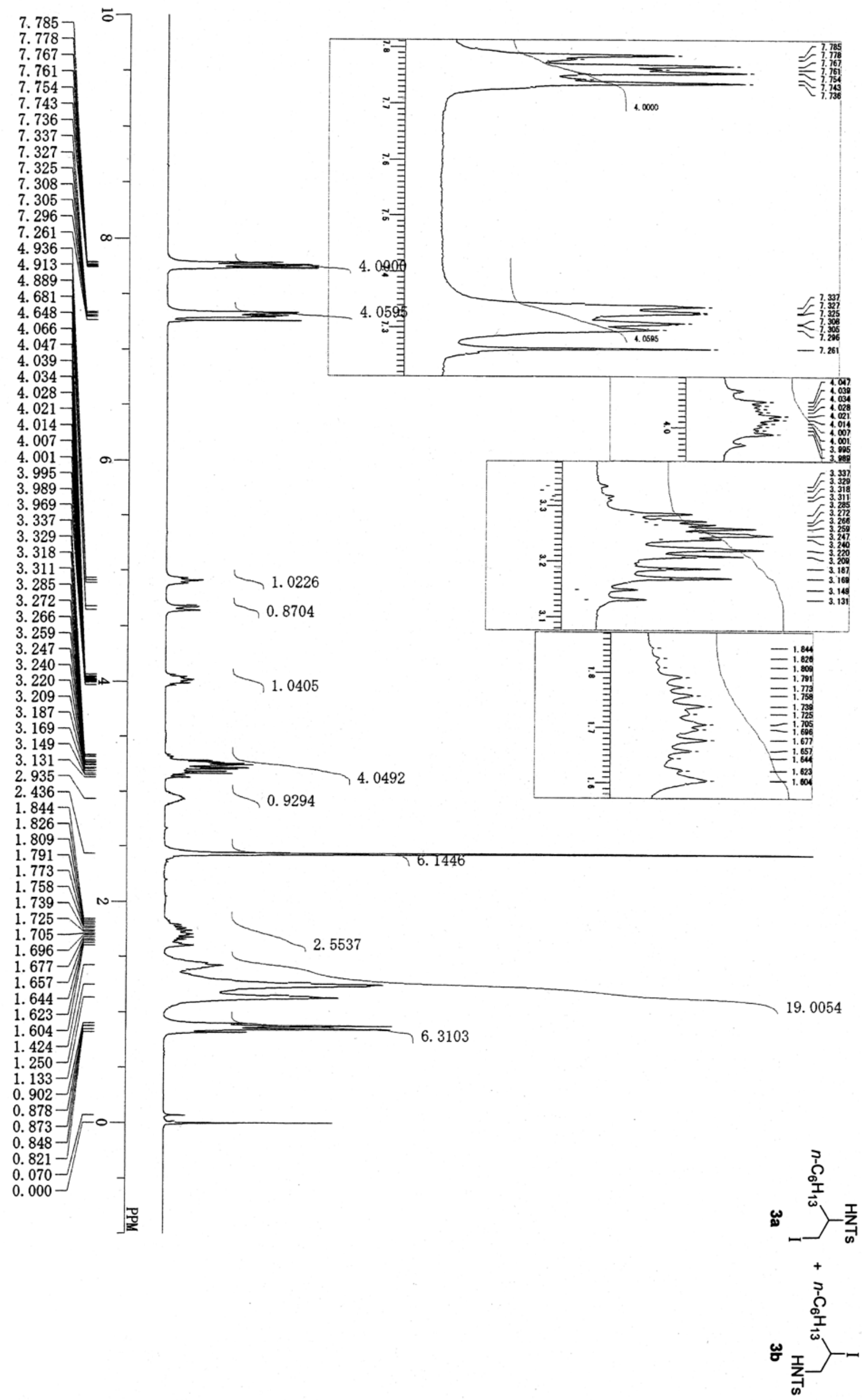




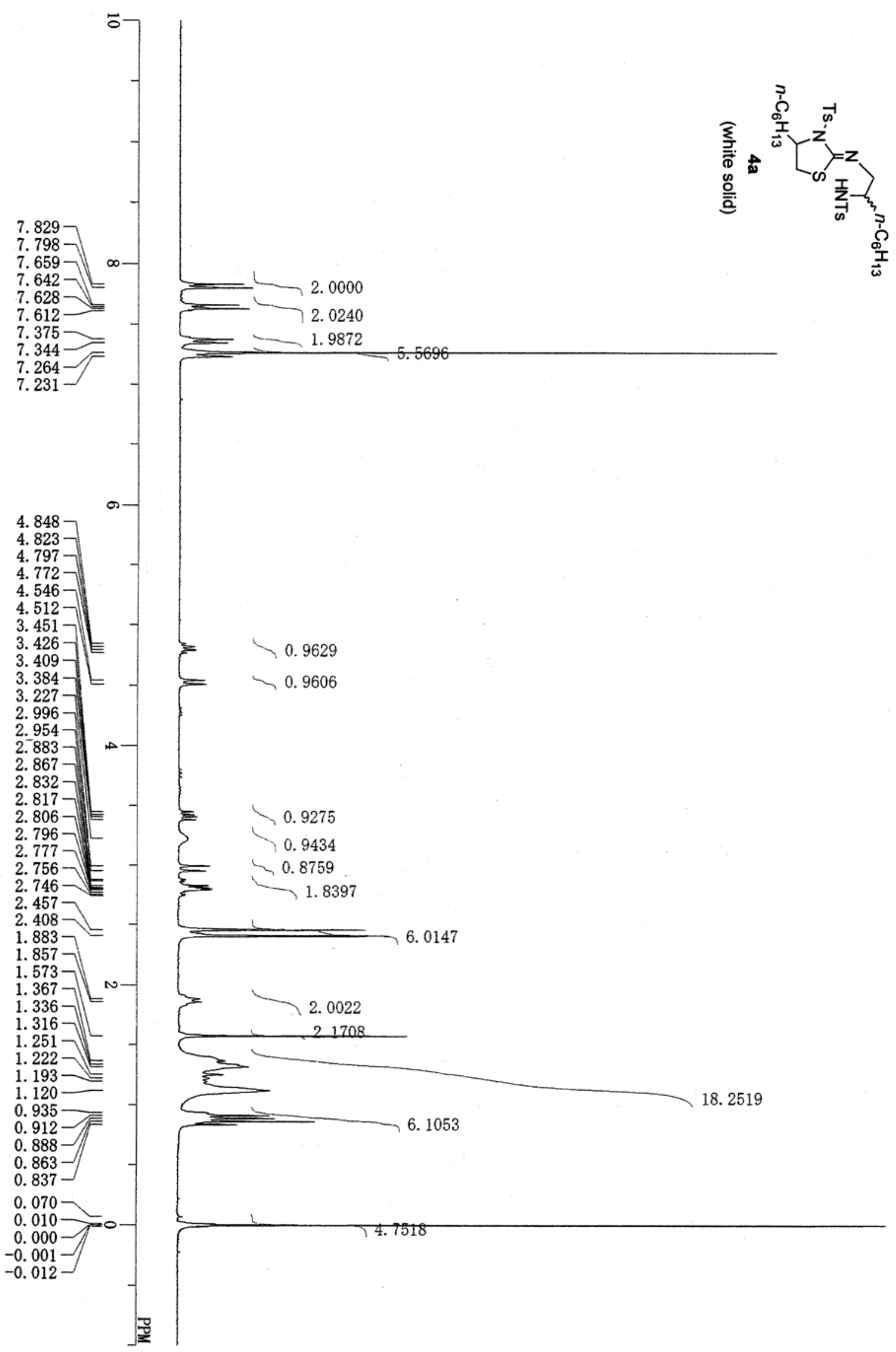



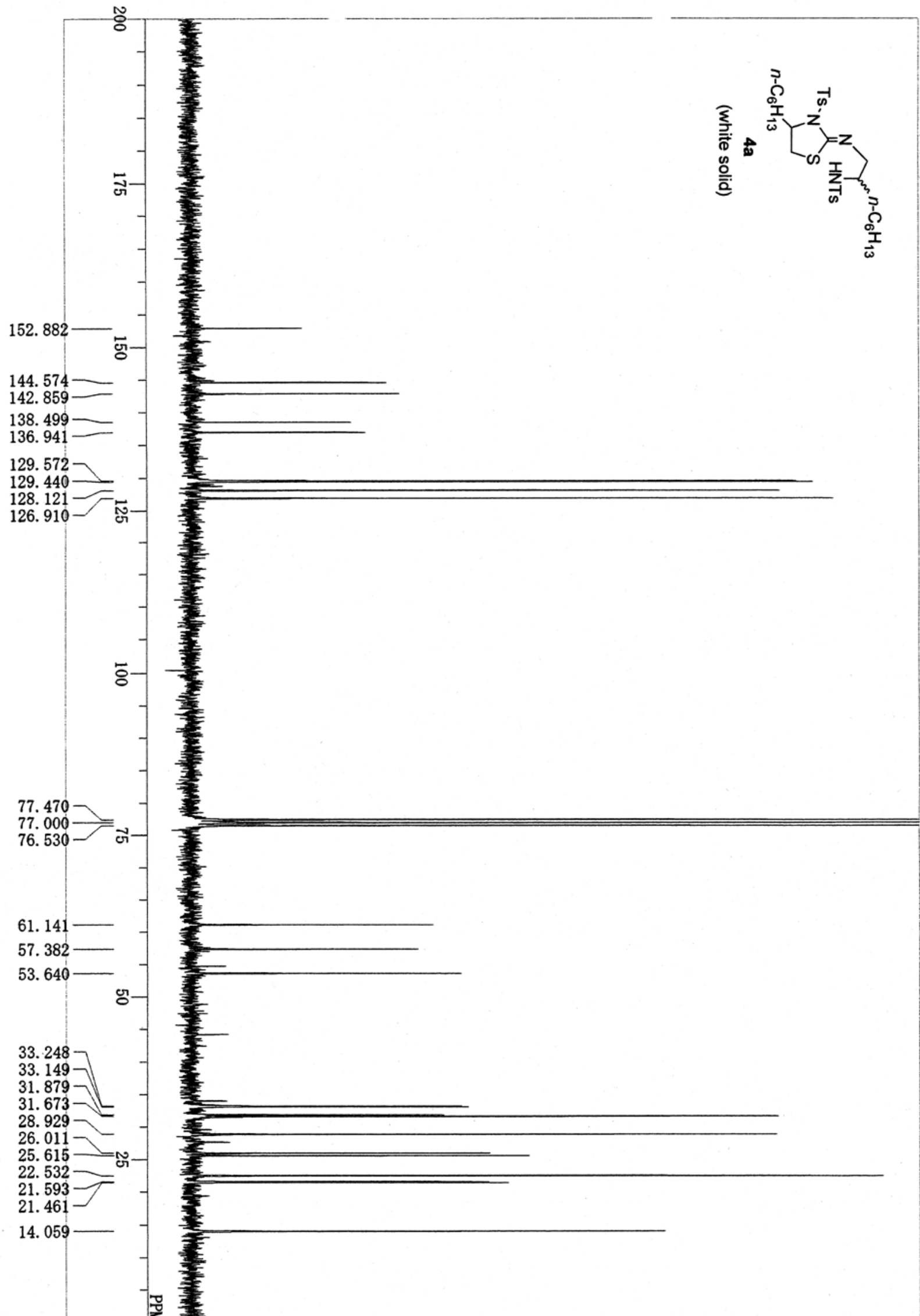

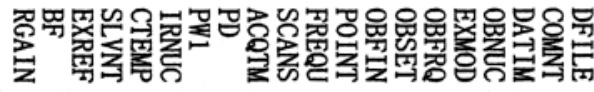

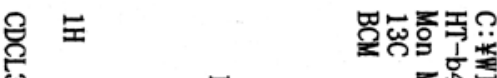

- N v r.t.

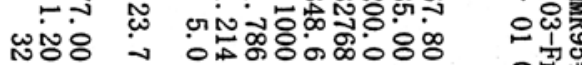

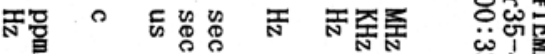

फ़ 


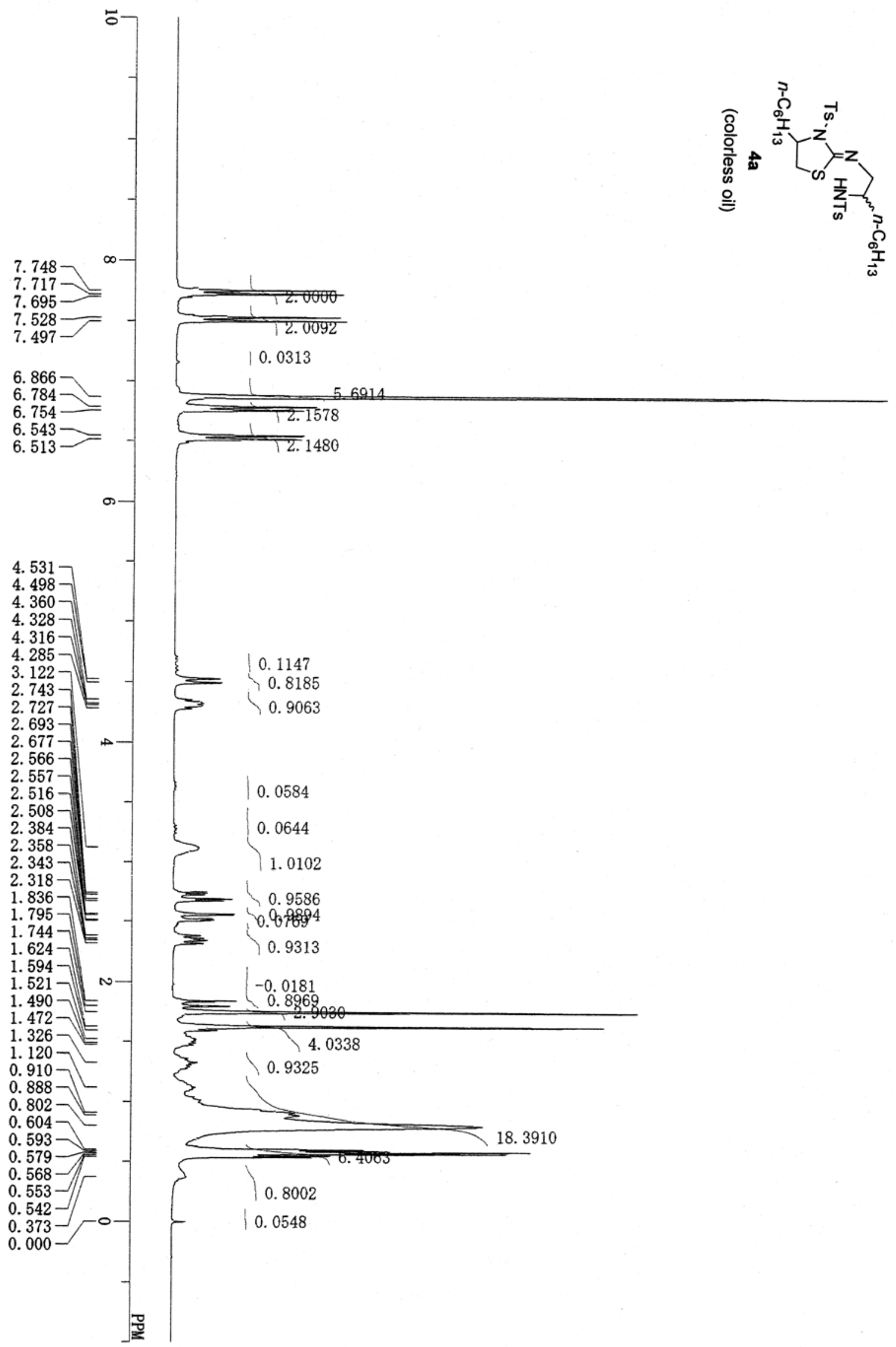



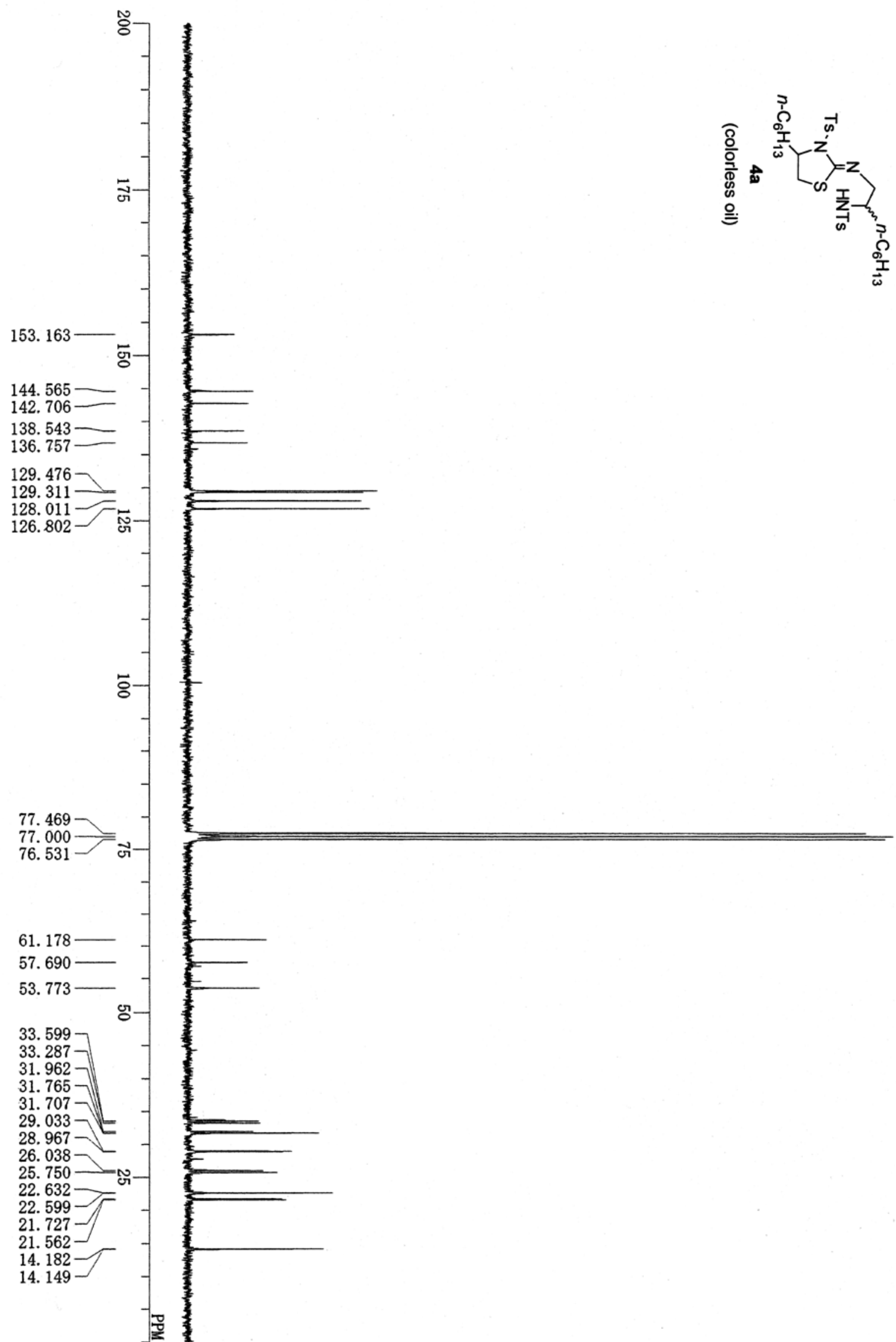